\title{
Proposed Spontaneous Generation of Magnetic Fields by Curved Layers of a Chiral Superconductor
}

\author{
T. Kvorning, ${ }^{1}$ T. H. Hansson, ${ }^{1,2}$ A. Quelle, ${ }^{3}$ and C. Morais Smith ${ }^{3}$ \\ ${ }^{1}$ Department of Physics, Stockholm University, AlbaNova University Center, SE-106 91 Stockholm, Sweden \\ ${ }^{2}$ Nordita, KTH Royal Institute of Technology and Stockholm University, Roslagstullsbacken 23, SE-106 91 Stockholm, Sweden \\ ${ }^{3}$ Institute for Theoretical Physics, Center for Extreme Matter and Emergent Phenomena, \\ Utrecht University, Princetonplein 5, 3584CC Utrecht, Netherlands
}

(Received 20 September 2017; published 25 May 2018)

\begin{abstract}
We demonstrate that two-dimensional chiral superconductors on curved surfaces spontaneously develop magnetic flux. This geometric Meissner effect provides an unequivocal signature of chiral superconductivity, which could be observed in layered materials under stress. We also employ the effect to explain some puzzling questions related to the location of zero-energy Majorana modes.
\end{abstract}

DOI: 10.1103/PhysRevLett.120.217002

Introduction.-Although it has been known for quite a while that all (gapped) superconductors are topologically ordered (see e.g., [1]), the chiral ones are particularly fascinating. Most interesting are the odd-pairing chiral superconductors $(\chi \mathrm{SCs})$ in two spatial dimensions $(2 d)$, and the layered ones in $3 d$, such as chiral $p$-wave, $f$-wave, etc.. Typically, these states support vortices that are nonAbelian anyons [2-5].

There are several candidate materials for chiral pairing and most of these are layered. Examples range from $\mathrm{UPt}_{3}$ [6], $\mathrm{Li}_{2} \mathrm{Pt}_{3} \mathrm{~B}$ [7], and $\mathrm{Sr}_{2} \mathrm{RuO}_{4}$ [8,9] for odd pairing, to SrPtAs $[10,11]$ and doped graphene [12-16] for even pairing.

Most of the experimental evidence for $\chi \mathrm{SC}$ is by observation of spontaneous breaking of time-reversal invariance, but the experiments are inconclusive and it is essential to find an unequivocal signature for $\chi \mathrm{SCs}$, similar to the Meissner effect in ordinary SCs. Since the essence of the Meissner effect is the gap to flux excitations, one can think of a SC as a flux insulator. Ordinary charge insulators can be either trivial or topological, so it is natural to ask whether the proper description of topological SCs would be in terms of topological flux insulators.

In this Letter, we show that a $2 d \chi \mathrm{SC}$ will spontaneously develop a magnetic flux when put on a curved surface. Conversely, if a spontaneously generated magnetic field is observed, the very fact that one of the two directions perpendicular to the surface is picked out clearly shows that there are supercurrents breaking chirality. We thus submit that the geometric Meissner effect, i.e., the spontaneous magnetic field due to curvature, will be a smoking-gun signature of a layered $\chi \mathrm{SC}$.

To understand this effect, it is useful to recall that in addition to the Hall conductivity, quantum Hall $(\mathrm{QH})$ liquids are characterized by their response to the curvature of the $2 d$ surface on which they reside. This effect, which was first described by Wen and Zee [17], comes about because an electron in a QH liquid carries a "spin" due to the cyclotron motion (often referred to as orbital spin), and thus acquires a Berry phase when moving on a curved surface. When completing a closed orbit on a surface with constant Gaussian curvature $K$ and magnetic field $B$, it will pick up a phase $\sim$ Area $\times(e B+s K)$, where $s$ is the orbital spin. Since the $\mathrm{QH}$ liquids form at high magnetic fields, the contribution from curvature cannot be detected in an experiment.

In a $\chi \mathrm{SC}$, the situation is very different. The conditions for detecting the magnetic flux response to curvature is much more favorable. Because there is no background flux, our results show that the geometric Meissner effect could be detected in a bent layered $\chi \mathrm{SC}$ using a sensitive superconducting quantum interference device (SQUID).

After a short review of the effective response theories for charge and flux insulators, we identify the origin of the geometric Meissner response and use this finding to resolve some puzzling questions related to the location of zeroenergy Majorana edge modes (Majorinos) and design a geometry-driven tunneling current in a weak link. Finally, we discuss possible experiments to detect our theoretical predictions.

Response action for $2 d U(1)$ insulators. - Insulators are systems with a conserved $U(1)$ charge and a gap to charged bulk excitations, implying that the response action is local. In the standard case of the $U(1)$ electromagnetic gauge symmetry related to electric-charge conservation, it is known that insulators can be trivial or topological. The simplest trivial insulator is just empty space, while others differ by having a more complicated electromagnetic response, with material-dependent parameters that can be continuously changed to those of the vacuum. A nontrivial, or topological, insulator cannot be continuously changed into the vacuum, and the effective action typically has terms with quantized coefficients that can change only at phase transitions related to the closing of the energy gap. Typical 
examples in $2 d$ are Chern insulators and integer $\mathrm{QH}$ systems. We first consider the known case of an electric insulator to exploit the analogy with flux insulators, i.e., superconductors, to which we then turn our attention.

(i) Charge insulators. Here, the effective action $W\left[A_{\mu}\right]$ encodes current correlation functions and the response to external electromagnetic fields $A_{\mu}$; i.e., the current expectation value is $\left\langle j_{\mu}\right\rangle=2 \pi \delta W / \delta A_{\mu}$. The $\mathrm{QH}$ response to a slowly varying current is encoded in the Chern-Simons (CS) term

$$
\begin{aligned}
W\left[A_{\mu}\right] & =W_{\mathrm{CS}}\left[A_{\mu}\right]+\cdots \\
& =\frac{\nu e^{2}}{2 h} \int d t d^{2} x \varepsilon^{\mu \nu \sigma} A_{\mu} \partial_{\nu} A_{\sigma}+\cdots
\end{aligned}
$$

which not only implies a Hall conductivity $\sigma_{H}=\nu e^{2} / h$, but also relates the total charge $N_{Q}$ of a region $S$ to the total flux $N_{\phi}$ through it. Changing the number of flux quanta $N_{\phi}$ by $\delta N_{\phi}$ will, according to (1), lead to a change $\delta N_{Q}=\nu \delta N_{\phi}$ in the number of unit charges $N_{Q}$. If the electromagnetic field is the only long-distance effect, which is the case in a pristine $\mathrm{QH}$ experiment, this relation also holds for the total values,

$$
N_{Q}=\nu N_{\phi} .
$$

Note that the sign of $\nu$ defines an orientation on the $2 d$ surface and thus breaks chiral symmetry.

We now turn to the main topic of this paper-the effect of geometry, i.e., how the system depends on a spatial (possibly time-dependent) metric $g_{i j}$. It was shown in Ref. [18] that the long-wavelength part of the geometric response is captured by the Wen-Zee term [17]

$$
W_{W Z}\left[A_{\mu}, \omega_{\mu}\right]=\frac{e \kappa_{Q H}}{2 \pi} \int d t d^{2} x \varepsilon^{\mu \nu \sigma} \omega_{\mu} \partial_{\nu} A_{\sigma},
$$

where $\omega_{\mu}$ (which depends on $g_{i j}$ ) is a potential for the Gauss curvature $K$, viz. $\varepsilon^{i j} \partial_{i} \omega_{j}=\sqrt{g} K$, and $\kappa_{Q H}$ defines the long wavelength charge response to the curvature. Just as the CS term, the Wen-Zee term specifies an orientation given by the sign of $\kappa_{Q H}$, so it can again only be present if there is a preferred orientation. For closed surfaces, the Wen-Zee term gives rise to a shift in the relation (2),

$$
N_{Q}=\nu N_{\phi}+\kappa_{Q H} \chi
$$

where $\chi=\int d^{2} x \sqrt{g} K / 2 \pi$ is the Euler characteristic of $S$. Since $N_{Q}, N_{\phi}$, and $\chi$ are integers, and $\nu$ is rational, $\kappa_{Q H}$ must be quantized.

(ii) Flux insulators. We now switch to the systems of interest - the $\chi$ SCs. In the spirit of Ref. [1], we will use a toy model where the electromagnetic field is mimicked by $2 d$ Maxwell theory. In $2 d$, conservation of magnetic flux (which is a consequence of Maxwell's equations)

$$
0=\partial_{\mu} j_{\text {flux }}^{\mu} \equiv \frac{1}{2} \partial_{\mu} \varepsilon^{\mu \nu \sigma} F_{\nu \sigma}
$$

amounts to having a conserved $U(1)$ charge. Since a $\mathrm{SC}$ is a flux insulator, i.e., has a gap to flux excitations, it is natural to consider the effective action $W\left[b_{\mu}\right]$, where the external gauge field $b_{\mu}$ is coupled to $j_{\text {flux }}^{\mu}$, so that $\left\langle j_{\text {flux }}^{\mu}\right\rangle=\delta W / \delta b_{\mu}$ is the expectation value of flux current. This coupling can be interpreted in two different ways, as seen by

$$
\begin{aligned}
\int d t d^{2} x j_{\text {flux }}^{\mu} b_{\mu} & =\int d t d^{2} x A_{\mu} \varepsilon^{\mu \nu \sigma} \partial_{\nu} b_{\sigma} \\
& =\int d t d^{2} x j^{\mu} A_{\mu}
\end{aligned}
$$

which identifies $\varepsilon^{\mu \nu \sigma} \partial_{\nu} b_{\sigma}$ as the supercurrent.

Ordinary SCs have chiral symmetry and are trivial flux insulators. However, $\chi \mathrm{SCs}$ could also be topologically nontrivial and we now focus on the response to curvature given by the SC version of the Wen-Zee term

$$
W_{W Z}\left[b_{\mu}, \omega_{\mu}\right]=\frac{\kappa_{C} \Phi_{0}}{2 \pi} \int d t d^{2} x \varepsilon^{\mu \nu \sigma} \omega_{\mu} \partial_{\nu} b_{\sigma},
$$

defined by the single parameter $\kappa_{C}$, which, just as $\kappa_{Q H}$, has to be quantized.

Equation (7) encodes the geometric Meissner response, which relates the total flux through a region $S$ to its total curvature $\chi$,

$$
N_{\Phi}=\kappa_{C} \chi
$$

$N_{\Phi}$ denotes flux in units of the superconducting flux quantum $\Phi_{0}=h / 2 e$. Changing the sign of $\kappa_{C}$ defines the direction of the magnetic field and it thus defines an orientation i.e., a chirality. Thus, only a chiral system can have a nonzero $\kappa_{C}$.

To see why we expect a nontrivial topological response, e.g. $\kappa_{C} \neq 0$, we consider a very thin film with small curvature, i.e. $K \xi^{2} \ll 1$, where $\xi$ is the size of the Cooper pair. Then, the orbital spin of the Cooper pair (i.e., the spin of the pair due to orbital motion) is well defined and perpendicular to the surface. If the orbital spin of the pairs all have the same chirality the pair will respond to curvature in a similar way as to a magnetic field. In addition to the Aharanov-Bohm phase due to the charge $2 e$ encircling the magnetic flux, the pair will also pick up the Berry phase $2 \pi \chi l$, where $l$ is the orbital spin of the pair (we take $l>0$ to denote right-handed rotation.) This means that the pair effectively responds to the combination of magnetic field and Gauss curvature, so that the Meissner effect will amount to expelling the combination $B+l K \Phi_{0} / 4 \pi$, rather than the magnetic field itself.

It is illuminating to see how the geometric Meissner effect emerges from a simple model, so we outline a 
derivation. The spatial part of the Wen-Zee response can be obtained from the Ginzburg-Landau free energy for a vector order parameter $\varphi$ describing $p$-wave paired spinless fermions. Mutatis mutandis, this model also applies to the spinfull case with half-vortices as described in Ref. [4]. The order parameter $\varphi$ can be written as

$$
\varphi=\sqrt{\rho_{+}} e^{i \theta_{+}}\left(\hat{e}_{1}+i \hat{e}_{2}\right)+\sqrt{\rho_{-}} e^{i \theta_{-}}\left(\hat{e}_{1}-i \hat{e}_{2}\right),
$$

where $\hat{e}_{1}$ and $\hat{e}_{2}$ are orthonormal basis vectors, and $\rho_{ \pm}$and $\theta_{ \pm}$are densities and phases of the two chiral components, respectively. We assume a Ginzburg-Landau free energy

$F=\int d^{2} x \sqrt{g}\left(\frac{\hbar^{2} g^{i j}}{2 m}\left(D_{i} \varphi\right)^{*} \cdot D_{j} \varphi+\frac{B^{2}}{2 \mu_{0}}+V(|\varphi|)\right)$,

where $m$ is a mass, $\mu_{0}$ is the magnetic permeability, $B=$ $1 / \sqrt{g} \varepsilon^{i j} \partial_{i} A_{j}$ is the magnetic field scalar, $i D_{i}=\left(i \partial_{i}-\right.$ $\left.2 e A_{i} / \hbar\right)$, and roman indices denote spatial coordinates. We take a potential $V(|\varphi|)$, for which there is a mean-field solution with $\bar{\rho}=\bar{\rho}_{+} \neq 0$ and $\bar{\rho}_{-}=0$. Such a potential must exist for the flat geometry in order to at all have a $\chi \mathrm{SC}$. By adiabatic continuity, such a solution will exist also for (at least weak) deformations of the surface, and since $\kappa_{C}$ is quantized, it will remain fixed as long as there is no phase transition.

To lowest order we then get the London free energy,

$F_{L}=\int d^{2} x \sqrt{g}\left(\frac{4 \hbar \bar{\rho}}{m}\left(\vec{\nabla} \theta_{+}+\vec{\omega}-\frac{2 e}{\hbar} \vec{A}\right)^{2}+\frac{B^{2}}{2 \mu_{0}}\right)$.

The square of the vector within the parenthesis is determined by the metric $g$, and $\vec{\omega}$ comes from the derivatives of the basis vectors $\hat{e}_{i}$. Varying $F_{L}$ gives

$$
\left(\lambda_{L}^{2} \triangle-1\right) B=\frac{\Phi_{0}}{4 \pi} K,
$$

where $\lambda_{L}=\sqrt{m /\left(32 \bar{\rho} \mu_{0} e^{2}\right)}$ is the London length and $\triangle$ is the Laplace operator defined by the metric $g$. For a region with a linear size much larger than $\lambda_{L}$, we can average both sides to get Eq. (8) with $\kappa_{C}=1$. Chiral pairing in the $l$ th channel would give $\kappa_{C}=l$. Note that this simple derivation does not give any flux-Hall response (see Ref. [19]) term in the effective action.

Thought experiments.-Some apparently puzzling issues can be understood in terms of the geometric Meissner effect.

(i) Where are the Majorino edge modes? A $\chi \mathrm{SC}$ generically has gapless edge modes in the thermodynamic limit, but odd pairing $\chi \mathrm{SCs}$ can support zero-energy Majorana edge modes (Majorinos), i.e., an exact zeroenergy mode for finite edge length (up to exponential corrections in system size).
For simple chiral $p$-wave SC models (such as in Ref. [2]) the zero-flux state on the cylinder supports edge Majorinos and the state with a flux quantum through the cylinder has no Majorinos. The opposite is true for the states on the annulus. Assuming an adiabatic change from the annulus to the cylinder, via a tipless cone, the SC should remain in its ground state. That would mean that Majorinos are either created or annihilated, which would imply a closing of the bulk gap which, in turn, would contradict the assumption of adiabaticity. In Ref. [20], we resolved this puzzle by showing that there is a level crossing and that the final state is not the ground state. We now show that this is easily understood as a geometric Meissner effect.

Since the surface of a tipless cone is flat, one might think that there would be no geometric Meissner effect. But $W_{W Z}$ in (7) depends on $\omega$, not on $K$, and the line integrals $\int \omega_{i} d x^{i}$ are nontrivial. With $\kappa_{C}=1$, the Wen-Zee term dictates that this geometric monodromy will be canceled by a flux through the hole of the cone. Going adiabatically from a cylinder to a disc amounts to the spontaneous creation of a flux, and the edge Majorinos will remain. To stay in the ground state by an avoided crossing would require the tunneling of a vortex across the $\mathrm{SC}$, which is exponentially suppressed in the system size.

Alternatively, one can interpolate between a cylinder and an annulus by gradually lifting the inner edge of the annulus to form part of a cylinder and an associated region with total curvature $\int d^{2} x \sqrt{g} K=-2 \pi$. When the cylindrical region is longer than $\lambda_{L}$, it follows from Eq. (12) that there will be a full flux quantum through the curved region. Since flux is conserved, it has to enter somewhere and if the system is large, it must have come from the inner edge, since tunneling from the outer edge is suppressed. This is shown on the left side of Fig. 1, where the surface is embedded in $3 d$ space and the strength and sign of the $2 d$ flux is illustrated by a $3 d$ field configuration (this configuration would be qualitatively correct for layered $3 d$ films, but only if they are much thicker than $\lambda_{L}$ ). The flux lines are closing through the hole of the newly formed cylinder, such
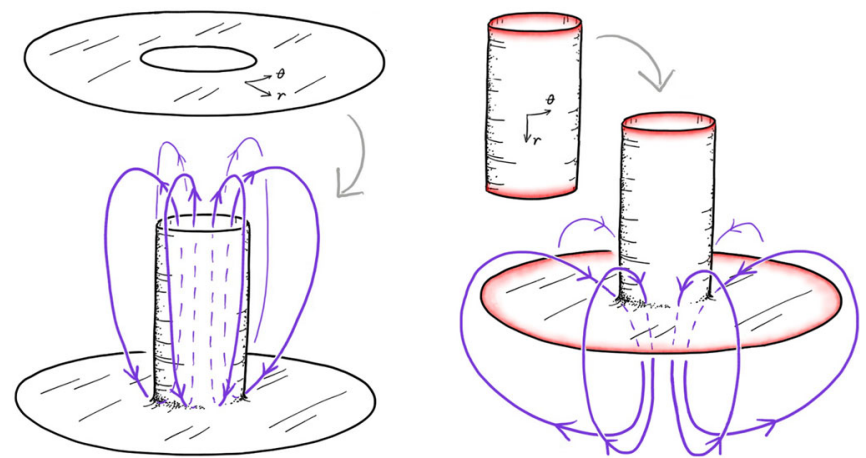

FIG. 1. Left: lifting the inner circle of an annulus to form a cylinder. Right: flattening the lower end of a cylinder to form an annulus. (Red indicates Majorino modes.) 
that the cylinder edge encircles a flux quantum while the annulus edge does not. Neither of them support edge Majorinos, just as the annulus we started from. If we do the opposite, i.e., flatten one end of a cylinder, we end up on the state shown in the right side of Fig. 1, that does support edge Majorinos.

Another perplexing question is what happens to the edge Majorino at the bottom of a cylinder, if the top is smoothly capped. The resulting state has only one edge, so there should be no Majorinos. But how can the bottom Majorino be removed by just a local change at the other end? Again, we can understand what happens by evoking the geometric Meissner effect. When we slowly deform an end of the cylinder to a half sphere, we create curvature and thus flux. If the cylinder is long enough, the flux must escape through the hole that we are about to close. In the limit of a very small hole we do not get a homogeneous flux on the half sphere, but a vortex, and thus a localized Majorino, as illustrated on the left side of Fig. 2. If we instead start from a sphere, and stretch one end out to form a cylinder, we end up in the flux configuration shown in the middle of Fig. 2a state with no Majorinos.

(ii) Closed manifolds. If we close both ends of the cylinder, as on the right in Fig. 2, vortices arise. We can deform this geometry by shrinking the cylindrical section to zero to get a sphere. In this case, there is no symmetry to give preferred locations to the vortices, but since vortices in a type II superconductor repel, they would sit at antipodal points to minimize energy. Picking a direction of the line between them amounts to a necessary spontaneous breaking of rotational symmetry.

That a $\chi \mathrm{SC}$ on a sphere must have vorticity is an effect analogous to the shift in the relation (4) between flux and charge in $\mathrm{QH}$ liquids. Here, it means that the number of flux quanta through a closed surface equals the integrated curvature $\chi$.

(iii) The geometric Josephson effect. Figure 3 shows how a cut cone can be formed by rolling up a segment cut from a Corbino disc. Since the geometry is flat, the ground state of
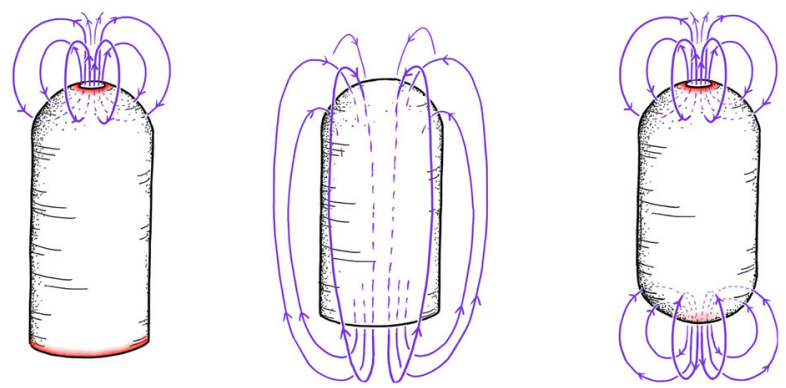

FIG. 2. Left: starting from a cylinder and closing one of the holes gives a magnetic flux at the cap. Middle: starting from a half-sphere and extending it, the flux points the opposite way, and extends all over the surface. This is not the ground state of the system due to the extended magnetic field lines. Right: as on the left, but closing both holes. (Red indicates Majorino modes.) the segment supports no flux, and neither does the partially rolled up configuration shown to the right. But the cut cone, obtained by gluing the disc along the dotted line, does support edge currents. What happens is that when the edges come close to each other, the system should be thought of as a superconductor with a weak link that can maintain a phase difference. As seen from Eq. (11), $\omega$ enters just as an electromagnetic vector potential giving a geometric version of Josephson effect.

(iv) The geometric flux pump. Laughlin notion of flux insertion in a Corbino geometry [21] was historically very important for understanding the integer quantization of the Hall conductance. In the QH case, a unit electric charge is pumped from one edge to the other by inserting a flux, and in the present context there is an analogous effect, which shows that the Wen-Zee term must be quantized. The geometric flux pump is operated by adiabatically transforming a cylinder into an annulus and then back to the cylinder by pulling one of the edges through the other, which has the net effect of turning the cylinder inside out. In this process, the initial and final states have the same Hamiltonian, so if it is adiabatic, the final state must be an energy eigenstate below the bulk gap. This implies that there is an integer number of superconducting flux quanta through the hole of the cylinder, which means that $\kappa_{C}$ must be quantized as an integer.

Experimental realizations. - Can the geometric Meissner effect be observed in the laboratory? Given a candidate $\chi \mathrm{SC}$, one can imagine several different experiments, depending on the material to be tested. Interesting candidates are $\mathrm{SrRuO}_{4}$ and bilayer graphene intercalated with $\mathrm{Ca}$, and to probe the symmetry of their order parameter one needs to grow them on a concave or convex substrate. For bilayers, it is required that the substrate on which the graphene is deposited must be nonsuperconducting, otherwise it will short-circuit the graphene, and destroy the geometric Meissner effect. Therefore, one could also conceive to suspend it on top of nanopillars, as already experimentally realized. However, here it would be convenient to have the nanopillars forming a circular array of a radius $R$, instead of a regular lattice, as in Ref. [22]. If the sample is much larger than the diameter of the circle, and can be anchored outside, we expect a downward curvature in the inner region of the circle, simply due to gravity. Another possibility would be to set up a standing wave in a

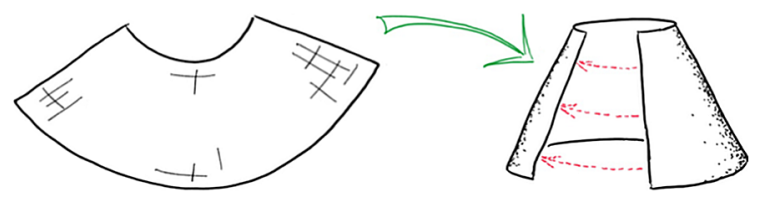

FIG. 3. Folding a $\chi \mathrm{SC}$ and creating a monodromy of the curvature form, results in a phase difference, and thus a current. The red arrows indicate the tunneling current. 
suspended sample and detect the ac electromagnetic response.

If the sample is a $2 d$ sheet thinner than the London length $\lambda_{L}$, the screening of charges and fluxes changes from exponential to a power law at large distances. Since we still have Meissner-like decay of the magnetic field, we would expect the relation (12) still to hold for regions with a radius $r \gg \lambda_{L}$, with corrections of order $1 / r$.

Comparing Eqs. (4) and (8), we see that in the QH case the geometric contribution to the flux is a small correction to the large dominant term due to the background magnetic field, while in the $\chi \mathrm{SC}$ case the geometric term stands alone.

The geometric Meissner effect scales proportional to the maximum bond-length stretching and inversely proportional to $\lambda_{L}^{2}$. With a maximum allowed bond-length stretching of $1 \%$ and $\lambda_{L}=1 \mu \mathrm{m}$, the magnetic field strength is of the order of $10 \mu \mathrm{T}$. The best SQUIDs can detect fields as small as a pT; hence, such a field should easily be detectable.

We hope that our work will motivate further experiments on curved $\chi \mathrm{SC}$ candidates, and contribute to the unveiling of this elusive state of matter in an unequivocal and definitive way.

We thank O. Golan, D. Mross, and S. Moroz for helpful discussions and and Sören Holst for all the nice hand-drawn pictures. The work by A.Q. and C.M.S. is part of the D-ITP consortium, a program of the Netherlands Organisation for Scientific Research (NWO) that is funded by the Dutch Ministry of Education, Culture and Science (OCW).

[1] T. Hansson, V. Oganesyan, and S. Sondhi, Ann. Phys. (Amsterdam) 313, 497 (2004).

[2] N. Read and D. Green, Phys. Rev. B 61, 10267 (2000).

[3] M. Greiter, X. Wen, and F. Wilczek, Nucl. Phys. B374, 567 (1992).
[4] D. A. Ivanov, Phys. Rev. Lett. 86, 268 (2001).

[5] A. Stern, F. von Oppen, and E. Mariani, Phys. Rev. B 70, 205338 (2004).

[6] H. Tou, Y. Kitaoka, K. Ishida, K. Asayama, N. Kimura, Y. Onuki, E. Yamamoto, Y. Haga, and K. Maezawa, Phys. Rev. Lett. 80, 3129 (1998).

[7] M. Nishiyama, Y. Inada, and G.-q. Zheng, Phys. Rev. Lett. 98, 047002 (2007).

[8] Y. Maeno, H. Hashimoto, K. Yoshida, S. Nishizaki, T. Fujita, J. Bednorz, and F. Lichtenberg, Nature (London) 372, 532 (1994).

[9] A. P. Mackenzie, T. Scaffidi, C. W. Hicks, and Y. Maeno, arXiv:1706.01942.

[10] M. H. Fischer, T. Neupert, C. Platt, A. P. Schnyder, W. Hanke, J. Goryo, R. Thomale, and M. Sigrist, Phys. Rev. B 89, 020509 (2014).

[11] Y. Nishikubo, K. Kudo, and M. Nohara, J. Phys. Soc. Jpn. 80, 055002 (2011).

[12] M. L. Kiesel, C. Platt, W. Hanke, D. A. Abanin, and R. Thomale, Phys. Rev. B 86, 020507 (2012).

[13] A. M. Black-Schaffer and C. Honerkamp, J. Phys. Condens. Matter 26, 423201 (2014).

[14] R. Nandkishore, L. S. Levitov, and A. V. Chubukov, Nat. Phys. 8, 158 (2012).

[15] A. Fedorov, N. Verbitskiy, D. Haberer, C. Struzzi, L. Petaccia, D. Usachov, O. Vilkov, D. Vyalikh, J. Fink, M. Knupfer, B. Büchner, and A. Grüneis, Nat. Commun. 5, 3257 (2014).

[16] S. Ichinokura, K. Sugawara, A. Takayama, T. Takahashi, and S. Hasegawa, ACS Nano 10, 2761 (2016).

[17] X. G. Wen and A. Zee, Phys. Rev. Lett. 69, 953 (1992).

[18] C. Hoyos and D. T. Son, Phys. Rev. Lett. 108, 066805 (2012).

[19] S. Moroz, A. Prem, V. Gurarie, and L. Radzihovsky, Phys. Rev. B 95, 014508 (2017).

[20] A. Quelle, C. M. Smith, T. Kvorning, and T. H. Hansson, Phys. Rev. B 94, 125137 (2016).

[21] R. B. Laughlin, Phys. Rev. B 23, 5632 (1981).

[22] M. A. Otte, V. Solis-Tinoco, P. Prieto, X. Borrisé, L. M. Lechuga, M. U. González, and B. Sepulveda, Small 11, 4201 (2015). 